\title{
EDITORIAL
}

\section{New year wish and updates}

\author{
Hypertension Research (2012) 35, 1-3; doi:10.1038/hr.2011.207
}

irstly, I would like to wish you all a very happy, prosperous new
year. In 2011, the 3rd year of Hypertension Research in its current format and platform (for online content), we again were kept busy working to further grow this journal. We have focused more on the 'everyday workings' of the journal, trying to be more selective and efficient in reviewing papers, in order to further improve the quality of the content. Some of these improvements have more to do with our work procedures and so may not be very visible to our readers. Herein, I would like to introduce some of these changes that were made in 2011.

Since 2009, the number of submissions to Hypertension Research has been increasing and we are trying hard to maintain a constant rate for processing papers to avoid delay. Currently, all received manuscripts are screened by our executive editors, and only papers that pass their check are sent to the responsible Associate Editor for the peer-review process. From the beginning of 2011, we have incorporated a more stringent set of requirements that we apply 'in-house' for newly submitted manuscripts. This has meant that only papers that we judge to be of interest to the community are being sent out for the peer-review process. This has enabled both a reduction in the time for decision and an improvement in the quality of papers being published in HR. We wish to continue this in the coming years, so would ask our reviewers to apply stricter criteria when evaluating the papers.

In 2011, we welcomed Dr Ji-Guang Wang and Dr Chang G Park to the board of Associate Editors. Responding to the increase in submissions from Asian countries, I am glad that we now have these two very distinguished editors representing HR in their regions. I sincerely hope that we'll be able to receive more quality submissions from their territories, as well as from many other countries.

Our 'Review Series' continues to publish collected review articles. In 2011, we solicited high-quality papers in the areas of 'Role of oxidative stress in hypertension and cardiovascular diseases' and 'New aspects of the sympathetic nervous system in hypertension'. Many of these review articles were made available for free online and were widely promoted to draw attention. As a result, they were often found in the monthly 'top 10' most frequently accessed or downloaded articles list. I am sure that the Review Series has contributed significantly to the increase in readership. Since 2009 , the average monthly number of visits to HR website has increased by $60 \%$. I am keen to continue publishing highquality review articles in this 'Review Series' format. In 2012, we are hoping for feature reviews in

1. Gender difference in hypertension

2. Genetics in hypertension

3. Hypertension and aging

I am sure the review papers in these topics would be well received by the community. I would like to thank the Associate Editors who have helped to plan these Review Series and worked hard to solicit manuscripts.

I should also mention that Hypertension Research introduced an Open Access (OA) option from January 2011, and started to offer authors the choice of publishing their papers as an OA article, making it available for free online immediately after it is published. Many journals are now publishing papers with this OA option, and many new 'Full OA' journals are being launched. This is certainly a new model of publishing that has been well received and demanded by the members of the scholarly community, including researchers in hypertension studies.

There are many items on our 'to do' list already for 2012, to further establish HR as a well-respected journal in the community. I would like to spend this year working to get these things done, although continuing to work on incoming manuscripts to make Hypertension Research a more mature and sophisticated journal with a truly international standard. I am lucky that I can work with my great colleagues and friends in the editorial board. With their great cooperation and help, I am hoping that we will see some good results from our editorial work at the end of this year.

Finally in closing, I'd like to acknowledge those reviewers who helped us in reading manuscripts and making decisions during 2011. Hypertension Research is fully reliant on their expert knowledge and warm support. The following individuals provided reviews of papers submitted to Hypertension Research from 1 October 2010 to 30 September 2011:

\section{Masatsugu Horiuchi Editor-in-Chief Hypertension Research}

\author{
Bakris, George \\ Banday, Anees \\ Banfi, Giuseppe \\ Barger, Mary \\ Barrios, Vivencio \\ Baulmann, Johannes \\ Baumann, Marcus \\ Baumert, Mathias
}


Bayley, JP

Baynard, Tracy

Bechara, Luiz Roberto Grassman

Belin De Chantemele, Eric Jacques

Bendhack, Lusiane

Ben-Dov, Iddo

Biaggioni, Italo

Bilo, Grzegorz

Blankesteijn, W Matthijs

Bobadilla, Norma

Bogatkevich, Galina

Borghi, Claudio

Bramlage, Peter

Brixner, Diana

Bruno, Rosa

Buck, Hudson

Bujold, Emmanuel

Burnier, Michel

Burti, Juliana

Buscemi, Silvio

Cameron, James

Casarini, Dulce

Casiglia, Edoardo

Cesana, Giancarlo

Chambers, Rachel

Chen, Chen-Huan

Chen, Jaw-Wen

Cheng, Xian Wu

Cheng, Zixi

Cho, Kyung Woo

Chung, Chang-Min

Cicero, Arrigo

Cornelissen, $\mathrm{V}$

Coto, Eliecer

Cox, Kay

Cruickshank, Kennedy

Cusi, Daniele

Daimon, Masao

Dandapat, Abhijit

Das, Undurti

De Leeuw, Peter

De Souza, Fabio

Derosa, Giuseppe

Di Iorio, Biagio

Dixon, Ian

Dohi, Yasuaki

Dolan, Eamon

Dominiczak, Anna

Dong, Yanbin

Dragun, Duska

Dunn, William

Eguchi, Kazuo

Elased, Khalid

Elsaie, Mohamed

Emoto, Noriaki

Erhardt, Peter

Evelyn, Torsney

Fazio, Giovanni

Ferro, Charles

Figueroa, Arturo

Fornage, Myriam
Franco, Martha

Freidin, Maxim

Fujisawa, Tomomi

Fujiwara, Yoshinori

Funder, John

Galetta, Fabio

Gandley, Robin

Gavish, Benjamin

Ghamari-Langroudi, Masoud

Giles, Thomas

Glowinska-Olszewska, Barbara

Gondim, Francisco

Gosse, Philippe

Granado, Nisara

Granger, Neil

Griendling, Kathy

$\mathrm{Gu}$, Dongfeng

$\mathrm{Gu}$, Mingliang

Guo, Tingwei

Gupta, Alok

Gyselaers, Wilfried

Hagiwara, Hiromi

Han, Seung Hyeok

Hansen, Tine

Hasebe, Naoyuki

Hashimoto, Junichiro

Hata, Kenichiro

Hayashi, Koichi

Hayashi, Terumasa

Hazell, Tom

Heagerty, Anthony

Heidland, August

Hermenegildo, Carlos

Hermida, Ramon

Higaki, Jitsuo

Higashi, Yukihito

Higashiyama, Aya

Hirawa, Nobuhito

Hirohito, Metoki

Hirooka, Yoshitaka

Hitomi, Hirofumi

Hocher, Berthold

Homma, Yoshimi

Horinaka, Shigeo

Horio, Takeshi

Hornum, Mads

Hoshide, Satoshi

Hosoya, Tatsuo

Hozawa, Atsushi

$\mathrm{Hu}$, Weichih

Hübner, Norbert

Hulver, Matthew

Ichihara, Atsuhiro

Ichiki, Toshihiro

Igase, Michiya

Ikari, Yuji

Imai, Enyu

Imanaka-Yoshida, Kyoko

Imanishi, Toshio

Inden, Yasuya

Inoue, Ituro
Inoue, Teruo

Inui, Naoki

Inukai, Kouichi

Irita, Jun

Irminger-Finger, Irmgard

Irwin, Richard

Iseki, Kunitoshi

Ishigami, Tomoaki

Ishikawa, Shizukiyo

Ishimitsu, Toshihiko

Isobe, Mitsuaki

Ito, Hiroshi

Ito, Masaaki

Ito, Masahiro

Ito, Satoru

Iwai, Masaru

Iwasaki, Yasumasa

Izawa, Hideo

Jeon, Byeong Hwa

Jialal, Ishwarlal

Jonas, Jost

Jordan, Jens

Juonala, Markus

Kadota, Aya

Kagiyama, Shuntaro

Kagota, Satomi

Kai, Hisashi

Kaji, Hiroshi

Kamide, Kei

Kamoi, Kyuzi

Kario, Kazuomi

Karumanchi, Ananth

Katabami, Takuyuki

Kato, Johji

Katsuya, Tomohiro

Kavey, Rae

Kawai, Hiroya

Kawamoto, Atsuhiko

Kawamoto, Ryuichi

Kawamura, Minoru

Kawamura, Takashi

Kawano, Yuhei

Kawasaki, Hiromu

Khalil, Asma

Khalil, Raouf

Khundmiri, Syed

Kikuya, Masahiro

Kim, Cheol-Ho

Kim, David

Kim, Deok Won

Kim, Soo Wan

Kimura, Genjiro

Kips, Jan

Kitakaze, Masafumi

Kitamura, Kazuo

Kitamura, Kenichiro

Kobayashi, Hiroshi

Kobayashi, Naohiko

Kobayashi, Shuzo

Kobori, Hiroyuki

Kohara, Katsuhiko
Kohler, Malcolm

Kokubo, Yoshihiro

Komai, Norio

Kondo, Takahisa

Konoshita, Tadashi

Konta, Tsuneo

Koshiyama, Hiroyuki

Kosuge, Masami

Kotani, Kazuhiko

Kotsis, Vasilios

Koya, Daisuke

Kreutz, Reinhold

Krzych, Lukasz

Kumagai, Hiroo

Kurata, Akira

Kurata, Mie

Kureishi-Bando, Yasuko

Kurtz, Theodore

Kusano, Eiji

Kushiro, Toshio

Kuwahara, Koichiro

Lee, Shin-Da

Leenen, Frans

Leeson, Paul

Lenz, Thomas

Li, Chunyan

Li, Jun

Liao, Ronglih

Lim, Se-Joong

Lindley, Richard

Lindsey, Merry

Litwin, Mieczyslaw

Liu, De-Pei

Lokhandwala, Mustafa

Lorenzen, Johan

Maeda, Seiji

Makino, Hirofumi

Mangge, Harald

Manolis, Athanasios

Mantero, Franco

Marti Del Moral, Amelia

Masugata, Hisashi

Masuo, Kazuko

Materson, Barry

Matsubara, Hiroaki

Matsubara, Tatsuaki

Matsui, Yoshio

Matsukawa, Kanji

Matsumura, Yasuo

Matsuoka, Hiroaki

Matsushita, Kunihiro

Matsuura, Hideo

Mceniery, Carmel

Mervaala, Eero

Miki, Tetsuro

Millis, Richard

Milovanovic, Branislav

Minamino, Tohru

Minatoguchi, Shinya

Mita, Mitsuo

Miura, Katsuyuki 
Miura, Shin-Ichiro

Miura, Tetsuji

Miyamori, Isamu

Miyano, Ichiro

Miyata, Masaaki

Modesti, Pietro Amedeo

Mogi, Masaki

Molvarec, Attila

Moreira, Leila

Morgan, Linda

Mori, Kiyoshi

Morimoto, Sachio

Morimoto, Satoshi

Morimoto, Shigeto

Morishita, Yoshiyuki

Moriya, Hidekazu

Mukoyama, Masashi

Müller, Gerhard

Munakata, Masanori

Murakami, Shougo

Nabika, Toru

Nagai, Michiaki

Nagao, Masaya

Nagata, Kohzo

Nagata, Masaharu

Nakagami, Hironori

Nakahashi, Takeshi

Nakamura, Kazuhiro

Nakamura, Koshi

Nakamura, Motoyuki

Nakamura, Tetsuya

Nakamura, Tsukasa

Nakamura, Yasuyuki

Nakano, Daisuke

Nakata, Yoshio

Nakata, Yukiko

Namdar, Mehdi

Narayanan, Damodaran

Narita, Ichiei

Natale, Francesco

Navar, L Gabriel

Ndao, Cheikh

Nguyen, Geneviève

Niccoli, Giampaolo

Ninomiya, Toshiharu

Nishikawa, Tetsuo

Nishikimi, Toshio

Nishiyama, Akira

Nitta, Kosaku

Node, Koichi

Noguchi, Katsuhiko

Numaguchi, Yasushi

Nussberger, Juerg

Ochiai, Ryuji

Ogata, Hiroaki

Ogawa, Hisao

Ogawa, Tetsuya

Ogimoto, Akiyosi

Ohashi, Naro

Ohishi, Mitsuru
Ohkubo, Takayoshi

Ohshima, Kousei

Ohya, Yusuke

Oka, Toru

Okada, Hirokazu

Okamura, Tomio

Okayama, Satoshi

Okumura, Kenji

Okura, Hiroyuki

Okuyama, Harumi

Onakpoya, Igho

Oparil, Suzanne

O'Rourke, Mf

Os, Ingrid

Otsuka, Fumio

Otsuka, Kuniaki

Ozono, Ryoji

Pacak, Karel

Padmanabhan, Sandosh

Padyukov, Leonid

Pagkalos, Michael

Paulis, Ludovit

Pavlik, Valory

Pérez-López, Faustino

Perman, Gaston

Perticone, Francesco

Pestana, Manuel

Petek-Ster, Marija

Peterlin, Borut

Phillips, Robert

Phisitkul, Sorot

Pope Iii, Arden

Pravenec, Michal

Prior, Steven

Procopciuc, Lucia

Prosser, Hamish

Quinn, Carol

Raasch, Walter

Rahmouni, Kamal

Reckelhoff, Jane

Reed, Shelby

Reusz, György

Riederer, Monika

Roberts, Mary

Robinson, Stephen

Ruilope, Luis

Ryuzaki, Munekazu

Sabio, José

Safar, Michel

Sagara, Miki

Saito, Ikuo

Saito, Isao

Saito, Yoshihiko

Sakata, Yasuhiko

Salles, Gil

Salvi, Paolo

Sanchez, Ramiro

Sano, Motoaki

Sansom, Steven

Santos, Raul
Sari, Ibrahim

Sasamura, Hiroyuki

Sata, Masataka

Sato, Akira

Sato, Atsuhisa

Sato, Yuichiro

Sato, Yuzo

Satoh, Minoru

Schalinske, Kevin

Schillaci, Giuseppe

Schmidt, Reinhold

Schteingart, David

Schultz, Harold

Schupp, Michael

Schutte, Rudolph

Segers, Patrick

Seki, Shingo

Sekizuka, Hiromitsu

Senbonmatsu, Takaaki

Shale, Dennis

Shaul, Philip

Shimosawa, Tatsuo

Shinichi, Oomama

Shiomi, Toshiaki

Shirai, Kohji

Silver, Robert

Skilton, Michael

Sookoian, Silvia

Sorlie, Paul

Spitsyn, Victor

Stergiou, George

Stompor, Tomasz

$\mathrm{Su}$, Jin-Bo

Sugimachi, Masaru

Sugimoto, Ken

Sugiyama, Atsushi

Sun, Wei

Tabara, Yasuharu

Taddei, Stefano

Takahashi, Masafumi

Takahashi, Wakoh

Takai, Shinji

Takase, Bonpei

Takazawa, Kenji

Takeda, Yasuharu

Takemura, Hirofumi

Takiuchi, Shin

Tamura, Kouichi

Tanaka, Hirofumi

Tao, Jun

Terada, Seishi

Terada, Yoshio

Tojo, Akihiro

Tokudome, Takeshi

Tomita, Naruya

Tomiyama, Hirofumi

Tomlinson, Brian

Tóth, Miklós

Townsend, Raymond

Toyoda, Kazunori
Tranquilli, Andrea

Tsuchihashi, Takuya

Tsuchihashi-Makaya, Miyuki

Tsuda, Kazushi

Tsuji, Yukiomi

Tsujino, Takeshi

Tsukimori, Kiyomi

Tsutsui, Hiroyuki

Ueda, Seiji

Ueno, Takahiro

Ulasi, Ifeoma

Umemoto, Seiji

Uzu, Takashi

Van Bortel, Luc

Van Vliet, Bruce

Vanderlei, Luiz Carlos

Veglio, Franco

Verdecchia, Paolo

Videla, Luis

Villegas, Raquel

Virdis, Agostino

Visseren, Frank

Vitarelli, Antonio

Wada, Manabu

Waki, Hidefumi

Walsh, Kenneth

Wang, Chunyu

Wang, Zuoguang

Watada, Hirotaka

Watanabe, Kenichi

Watanabe, Makoto

Weber, Thomas

Weinreich, Thomas

Wen, Xiaozhong

Wilkinson, Ian

Wilson, Sarah

Wilson, Thomas

Wyatt, Sharon

Wykretowicz, Andrzej

Xing, Dongqi

Yagi, Shusuke

Yamada, Yoshiji

Yamamoto, Kazuhiro

Yamamoto, Kenta

Yamasue, Kotaro

Yang, Wei-Shiung

Yano, Yuichiro

Yasuda, Osamu

Yasujima, Minoru

Yasukawa, Hideo

Yoshiaki, Taniyama

Yoshida, Masayuki

Yoshimoto, Takanobu

Yoshimura, Michihiro

Yoshiyama, Minoru

Yoshizumi, Masao

Zhang, Yuqing

Zhou, Xiyou

Zhu, Zhiming 